\title{
Are you even listening? A study of speakers' mimicry in non-response situations
}

\section{TANJA ALEXANDRA JUNGE}

This article focuses on the facial expressions of speakers in a situation where they are exposed to a non-responding listener. Four subjects participate in an experiment where they tell a personal story to a recipient instructed to abstain from reacting to what they are saying. By using Conversation Analysis and gesture research, it is shown how participants via facial resources attempt to achieve understanding with or without response from their recipients, and how it affects the story when this response fails to appear. A key finding is that the face and facial expressions emphasize the response seeking functions of voice, gaze and gesture. The analysis also shows that participants actively seek eye contact during important utterances. However, mutual gaze alone is not handled as sufficient proof by speakers that their talk is being heard or understood. The body and the face play a crucial role as meta-communicative ways for speakers to obtain the attention and understanding of their recipients. A general conclusion from the experiment is that attention cannot simply be equated with A's looking at B, and further, that the failure of listeners to mirror or in other ways show visual response has negative effects on speakers' storytelling.

KEYWORDS: facial expressions, non-response, Conversation Analysis, gesture, multimodality 